VOL. 9 (2005), 5-11

\title{
FIXED POINTS FOR NONSELF MAPPINGS IN METRIC SPACES
}

\author{
DAIGA GRUNDMANE
}

\begin{abstract}
In this paper we try to extend several well known fixed point theorems for nonself mappings in Banach spaces to mappings in metric spaces. To achieve this goal some additional requirements on convexity in metric spaces are needed. We introduce the notions of MP-convex and NMP-convex metric spaces and obtain several results on existence of fixed points for nonself nonexpansive mappings in NMP-convex metric spaces. In particular, the notion of weakly inward mappings is generalized for mappings in metric spaces and the existence of fixed points is proved for mappings satisfying this condition.
\end{abstract}

\section{InTRODUCTION}

The largest part of the known results on existence of fixed points for mappings in metric spaces are applicable for mappings $f: X \rightarrow X$, i.e. for mappings with equal domain and range. In [14] and [5] the author has been able to obtain several results on existence of fixed points also for nonself mappings in metric spaces. In these papers several generalizations of known fixed point theorems for strongly non-expansive and contractive mappings have been obtained by using a notion of reflector introduced in [14].

At the same time the problem of existence of fixed points for nonself mappings in Banach spaces has been widely studied and a large number of results has been obtained. Most of these theorems can be proved by using the so called retraction mappings that satisfy certain properties and that allow to reduce the existence of fixed points for a nonself mapping $f$ to existence of fixed points for some other self mapping $g$. In particular, in this way the existence of fixed points for interesting class of weakly inward mappings that satisfy some additional properties can be proved.

In this paper the author attempts to generalize several known results on existence of fixed points for nonself mappings in Banach spaces to nonself mappings in metric spaces. The obtained results show that there are many problems that makes such generalizations difficult. At the same time they also show that interesting generalizations in this direction in principle are possible and suggests that this can be a perspective area for further research.

1991 Mathematics Subject Classification. Primary 54H25. 


\section{Convexity in Metric SPACES}

An important role in fixed point theory for Banach spaces have mappings that are defined in convex sets. If we want to obtain similar results in metric spaces the first problem we have to deal with is how to define convexity in general metric space. Whilst in principle it can be done similarly as in Banach spaces by requiring that for each $x, y \in A$, where $A$ - convex set, and for each $z$ with $d(x, z)+d(z, y)=$ $d(x, y)$ we must have $z \in A$, such requirement will be too weak for most of practical purposes, since in general the structure of metric spaces does not require that there exist any $z$ with the property $d(x, z)+d(z, y)=d(x, y)$ and $z \neq x, z \neq y$. A known solution here could be to consider only so called strictly convex metric spaces introduced by W. Takahashi in [12] and recently studied also by I. Bula in [1] and [2]. (Note that the notion of strict convexity of Banach spaces differs from the notion of strict convexity of metric spaces that we introduce here.)

Definition 2.1. A metric space $X$ is strictly convex if for all $x, y \in X$ and all $t \in] 0,1[$ there exists a unique $z \in X$ such that $d(x, z)=t d(x, y)$ and $d(z, y)=$ $(1-t) d(x, y)$.

It is easy to see that in strictly convex metric spaces we can already speak about reasonable generalization of "line segment" connecting two distinct points $x$ and $y$. We denote such generalized "line segment" by $L(x, y)$ and formally define it by $L(x, y)=\{z \mid \exists t \in[0,1]: d(x, z)=t d(x, y) \& d(z, y)=(1-t) d(x, y)\}$.

Definition 2.2. Let $X$ be a strictly convex metric space. $A \subseteq X$ is convex if for all $x, y \in A$ and for all $z \in L(x, y)$ we have $z \in A$.

However strictly convex metric spaces still can possess some strange and unpleasant properties, namely, it may turn out that closed balls (we define a closed ball $B(x, r)$ with center in point $x$ and radius $r \in \mathbf{R}_{+}$(where $\mathbf{R}_{+}$stands for real positive numbers) by $B(x, r)=\{y \mid d(x, y) \leq r\})$ not necessarily are convex sets. This problem can pose severe difficulties, since convexity of balls also is often assumed and used in fixed point theorems in Banach spaces. A solution could be to consider only such strictly convex metric spaces in which all balls are convex sets.

Another approach could be to look for some other natural properties that hold for convex sets in Banach spaces (and probably could be used as equivalent definitions of convexity) and to consider strictly convex metric spaces that satisfy some property of such kind. A good candidate for such property appears to be existence of so called metric projection.

Definition 2.3. Let $X$ be a metric space and let $A \subseteq X$. We say that a mapping $P: X \rightarrow A$ is metric projection from $X$ to $A$, if for all $x \in X$ and all $y \in A$, $y \neq P(x)$, we have $d(x, P(x))<d(x, y)$.

If $X$ is Banach space and $A$ is compact and convex, then there exists a metric projection from $X$ to $A$. Since the existence of metric projection is widely used also in fixed point theorems it could be useful to consider strictly convex metric 
spaces $X$ in which there exists a metric projection $P: X \rightarrow A$ for each compact convex set $A \subseteq X$. We will call such metric spaces MP-convex (metric projection convex).

Definition 2.4. Let $X$ be a strictly convex metric space. $X$ is MP-convex if for each compact convex set $A \subseteq X$ there exists a metric projection $P: X \rightarrow A$.

In [2] it is shown that if $X$ is strictly convex metric space such that all closed balls $B(x, r) \subseteq X$ are also (strictly) convex sets, then $X$ is MP-convex. By similar simple argument we can prove also the converse result.

Proposition 2.1. Let $X$ be MP-convex metric space. Then all balls $B(x, r) \subseteq X$ are strictly convex.

Proof. Let $B(x, r)$ be a ball contained in $X$. Let assume that $B(x, r)$ is not strictly convex. It is not hard to show (using the fact that strict convexity in essence guaranties also several natural "continuity" properties of metric space) that then there exist two points $a$ and $b$ on border of $B(x, r)$, such that none of the points of $L(a, b)$ is interior point of $B(x, r)$, i.e. there exist $a, b \in X$ such that $d(x, a)=d(x, b)=r$ and for all $c \in L(a, b)$ we have $d(x, c) \geq r$. Now, from the definition of convex sets it follows that $L(a, b)$ is convex, also, it is easy to show that $L(a, b)$ is compact. Therefore there exists metric projection $P: X \rightarrow L(a, b)$. But, either $P(x)=a$, then $d(x, P(x))=d(x, a)$, or $P(x)=b$, then $d(x, P(x))=d(x, b)$, or $P(x)=y, y \neq a, y \neq b$, then $d(x, P(x)) \geq d(x, a)=d(x, b)$, that contradicts to the fact that $P$ is a metric projection. Hence our assumption that there exists a ball $B(x, r) \subseteq X$ that is not strictly convex fails.

Thus the notion of MP-convex metric spaces coincide with the notion of strictly convex metric spaces with (strictly) convex round balls introduced in [2]. The author does not know whether there exist strictly convex metric spaces with convex (but not strictly convex) balls, however the existence also of such "pathological" spaces seems to be very likely. From Proposition 2.1 it is clear that if such spaces exist they will not be MP-convex.

Another interesting property of compact convex sets in Banach spaces is the following. Let $X$ be a Banach space, $A \subseteq X$ a compact convex set and $P: X \rightarrow A$ a metric projection. Then it can be shown that for all $x, y \in X$ we have $d(x, y) \geq$ $d(P(x), P(y))$. This property for us will be very important, therefore we will consider NMP-convex (nonexpansive metric projection convex) metric spaces.

Definition 2.5. Let $X$ be a strictly convex metric space. $X$ is NMP-convex if $X$ is MP-convex and for each compact convex set $A \subseteq X$, and a metric projection $P: X \rightarrow A$ we have $d(x, y) \geq d(P(x), P(y))$ for all $x, y \in X$.

It is an interesting open problem whether MP-convex metric spaces are also necessarily NMP-convex. Apparently NMP-convexity is quite strong requirement and it is unclear how (and whether) it could be obtained just from MP-convexity. At the same time MP-convexity still places strong restrictions on the structure of 
metric space and as far have prevented us to find an example of MP-convex and not NMP-convex space.

\section{FIXED POINTS FOR NONSELF MAPPINGS IN BANACH SPACES}

A popular way to prove the existence of fixed points for nonself mappings in Banach spaces is to use retraction mappings. A general idea is to construct for a given mapping $f$ another self mapping $g$ for which the existence of fixed point is guaranteed by some already known theorem and then to show that any fixed point of $g$ is also a fixed point of $f$. It is not surprising that a typical candidate for such known result that guarantees existence of fixed point for mapping $g$ is Schauder's theorem that guarantees existence of fixed point for any continuous mapping $g: A \rightarrow A$, if $A$ is non-empty, compact and convex set. The last part of the argument that shows that any fixed point of $g$ is also a fixed point of $f$ apparently places some additional requirements on $f$. In a very general result (Theorem 18.1 in [3], originally proved by H. Mönch in [10]) such additional requirement for mapping $f: A \rightarrow X$ (reformulated in our metric space notation) is existence of point $x \in A$ such that for all $y \in \delta A$ (where by $\delta A$ we denote border of $A$ ) point $y$ does not belong to $L(x, F(y))$. Many other known results (in particular using the requirement of weak inwardness that we will consider below) can be easily reduced to the Mönch theorem mentioned above.

If we will attempt to prove such or similar result in (MP-convex) metric spaces we will face two difficulties. The first will be the lack of equivalent of Schauder's theorem that guarantees existence of fixed points just for continuous mappings in metric spaces. Of course it seems very temptating to try to generalize Schauder's result for metric spaces. However as far nobody has succeeded in this direction and it seems to be very likely that MP-convexity will be too weak condition for such generalization to be possible. Thus, at the very beginning we are forced to consider stronger requirements on mapping $f$ than just continuity. Still interesting candidate here remains non-expansive mappings, i.e. such that $d(f(x), f(y)) \leq$ $d(x, y)$ for all $x, y$. Then instead of Schauder's theorem we could exploit for example a popular theorem of W.A. Kirk in [6] that, by the way, is generalized for MP-convex (in our terminology) metric spaces in [2].

Theorem 3.1 (I. Bula). Let $X$ be MP-convex metric space. Let $A \subseteq X$ be compact and convex set and let $f: A \rightarrow A$ be non-expansive mapping. Then $f$ has a fixed point in $A$.

The second difficulty that will now arise will be the task to construct for a given non-expansive mapping $f: A \rightarrow X$ a non-expansive mapping $g: A \rightarrow A$. Whilst it is comparatively easy, if we are given only continuousness of $f$ and we require only continuousness of $g$, situation becomes much more difficult if we replace continuousness by non-expansiveness. It is easy to verify that requirement used in Mönch theorem in general will not work for non-expansive mappings. The possible solution here is to consider NMP-convex spaces in which there is a simple 
natural requirement that guarantees the non-expansiveness of $g$. However, the question of sufficiently general and/or easy verifiable requirements remains.

\section{Results}

Our basic result is the following theorem that guarantees existence of fixed point for nonself mappings in NMP-convex metric spaces.

Theorem 4.1. Let $X$ be a NMP-convex metric space. Let $A \subseteq X$ be a compact and convex set and let $P: X \rightarrow A$ be a metric projection. Let $f: A \rightarrow X$ be non-expansive mapping such that for all $x \in \delta A$ we have $P(f(x)) \neq x$. Then $f$ has a fixed point in $A$.

Proof. We define $g: A \rightarrow A$ by $g(x)=P(f(x))$. Since $A$ is convex and $X$ is NMP-convex metric space we have $d(g(x), g(y))=d(P(f(x)), P(f(y))) \leq$ $d(f(x), f(y)) \leq d(x, y)$. Thus $g$ is non-expansive and by Theorem 3.1 there exists $x_{0} \in A$ such that $g\left(x_{0}\right)=x_{0}$. Now let assume that $f\left(x_{0}\right) \neq x_{0}$. Then $f\left(x_{0}\right) \notin A$ (because for all $x \in A$ with $f(x) \in A$ we have $g(x)=f(x))$, therefore $P\left(f\left(x_{0}\right)\right) \in$ $\delta A$. But then, by initial requirement on $f$, we have $P\left(f\left(x_{0}\right)\right)=g\left(x_{0}\right) \neq x_{0}$. Thus, $f\left(x_{0}\right)=g\left(x_{0}\right)$.

Unfortunately, the requirement that for all $x \in \delta A$ we must have $P(f(x)) \neq x$ seems to be quite strong and does not allow any flexibility in construction of mapping $g$. Of course, we can generalize this result by requiring that condition above holds for some convex compact subset $A^{\prime} \subseteq A$. The next result trivially follows from theorem 4.1.

Theorem 4.2. Let $X$ be a NMP-convex metric space. Let $A \subseteq X$ and let $f: A \rightarrow$ $X$ be a non-expansive mapping. If there exists $A^{\prime} \subseteq A$ such that $A^{\prime}$ is compact and convex and such that for all $x \in \delta A^{\prime}$ we have $P(f(x)) \neq x$, where $P: X \rightarrow A^{\prime}$ is metric projection, then $f$ has a fixed point in $A$.

Formulation of Theorem 4.2 allows more flexibility on construction of $g$, however the existence of appropriate set $A^{\prime}$ could be hard to verify. Also, it appears to be very likely that there still exist situations, in which it is easy to construct the mapping $g$ with required properties, but such mapping cannot be constructed by using just metric projection, and which therefore are not covered by Theorem 4.2. (An interesting, though probably still insufficiently general, situation could be the one in which $g$ is constructable via several metric projections. It is easy to see how to generalize Theorem 4.2 for such case, however such result apparently is too unpractical, and it still remains to be proved that several metric projections can not be substituted by a single projection to some other set.) The finding of more general (or different) ways how the appropriate function $g$ can be constructed appears to be an important topic for further research. Still, from Theorems 4.1 and 4.2 it is possible to obtain several corollaries that already uses quite familiar conditions on function $f$. 
Corollary 4.1. Let $X$ be a NMP-convex metric space such that all closed balls $B(x, r), x \in X, r \in \mathbf{R}_{+}$are compact sets. Let $x_{0} \in X, r \in \mathbf{R}_{+}$and let $f$ : $B\left(x_{0}, r\right) \rightarrow X$ be a mapping from a closed ball $B\left(x_{0}, r\right)$ to $X$ such that for all $y \in X$ with $d\left(x_{0}, y\right)=r$ condition $y \notin L\left(x_{0}, F(y)\right)$ holds. Then $f$ has a fixed point in $B\left(x_{0}, r\right)$.

Proof. Let $A=B\left(x_{0}, r\right)$. Let $P: X \rightarrow A$ be a metric projection. Consider a point $y \in X$ such that $d\left(x_{0}, y\right)=r$ (i.e. $y$ lies on border of $B\left(x_{0}, r\right)$ ). We can assume that $f(y) \neq y$ (otherwise the result automatically holds). If $f(y) \in B\left(x_{0}, r\right)$ then $P(f(y))=f(y) \neq y$. If $f(y) \notin B\left(x_{0}, r\right)$, then, due to strict convexity of $X$, there exists $z$ with $d\left(x_{0}, z\right)=r$ such that $z \in L\left(x_{0}, f(y)\right)$.

From our condition on $f$ we know that $z \neq y$. From strict convexity of $X$ we have that $d(f(y), y)>d(f(y), z)$. Thus, for all $y$ on border of $B\left(x_{0}, r\right)=A$ condition $P(f(y)) \neq f(y)$ holds. Hence the existence of fixed point follows from Theorem 4.1 .

An interesting and often considered condition that guarantees existence of fixed points for nonself mappings in Banach spaces is weak inwardness. In our terminology it can be defined in strictly convex metric spaces as follows.

Definition 4.1. Let $X$ be strictly convex metric space and let $A \subseteq X$. A mapping $f: A \rightarrow X$ is called weakly inward, if for all $x \in A$ with $x \neq f(x)$ there exists $y \in A$, such that $y \neq x$ and $y \in L(x, f(x))$.

Weak inwardness is considered to be a comparatively weak condition, therefore it may appear unexpected that our method, apparently weaker than methods available in Banach spaces, allows to prove the existence of fixed points for weaklyinward mappings.

Corollary 4.2. Let $X$ be a NMP-convex matric space and let $A \subseteq X$ be a compact convex set. Let $f: A \rightarrow X$ be weakly inward mapping. Then $f$ has a fixed point in $A$.

Proof. Consider metric projection $P: X \rightarrow A$. Let $y \in \delta A$. If $y=f(y)$ we are done. Otherwise, if $f(y) \in A$ we have $P(f(y))=f(y) \neq y$. If $f(y) \notin A$, then since $f$ if weakly-inward, there exists $z \in A$ such that $z \in L(y, f(y))$, hence $P(f(y)) \neq y$. Thus, either there exist fixed points on border of $A$, or for all $y \in \delta A$ we have $P(f(y)) \neq y$ and the existence of fixed point follows from theorem 4.1.

\section{REFERENCES}

[1] I. Bula. Fixed points for mappings in convex metric spaces, $\mathrm{PhD}$ thesis, The University of Latvia, 1994.

[2] I. Bula. Strictly convex metric spaces and fixed points, Mathematica Moravica, 3(1999), $5-16$.

[3] K. Deimling. Nonlinear functional analysis. Springer Verlag, 1985.

[4] Ky Fan. Some properties of convex sets related to fixed points theorems, Mathematische Annalen, 266(1984), 519-537. 
[5] D. Grundmane. Fixed points for nonself mappings, Acta Universitatis Latviensis, 606(1997), $37-44$.

[6] W.A. Kirk. An abstract fixed point theorem for nonexpansive mappings, Proceedings of American Mathematical Society, 82(1981), 640-642.

[7] W.A. Kirk. Fixed point theory for nonexpansive mappings, Lecture notes in mathematics, 886(1981), 484-505.

[8] J. Kulesza and T.C. Lim. On weak compactness and countable weak compactness in fixed point theory, Proceedings of the American Mathematical Society, 124(1996), 3345-3349.

[9] T.C. Lin. Approximations and fixed points for condensing non-self-maps defined on sphere, Proceedings of the American Mathematical Society, 105(1989), 66-69.

[10] K. Mönch. Boundary value problems for nonlinear ordinary differential equations of second order Banach spaces, Nonlinear Analysis, 4(1980), 985-999.

[11] S. Reich. Fixed points in locally convex spaces, Mathematische Zeitschrift, 125(1972), 17-31.

[12] V. Takahashi. A convexity in metric space and nonexpansive mappings I, Kodai Mathematical Seminar Reports, 22(1970), 142-149.

[13] Ts. Tsachev and V.G. Angelov. Fixed points of nonself-mappings and applications, Nonlinear analysis, 21(1993), 9-16.

[14] D. Zabarovska and A. Liepiņš. Mirrors and existence of fixed points for non-invariant mappings, Topological spaces and their mappings, (Acta Universitatis Latviensis), (1989), 88-92.

Faculty of Physics and Mathematics UNIVERSITY OF LATVIA

ZeĻL̇U iela 8, Rīga, LV - 1002, LATVia

E-mail address: daiga@nelss.Iv 